\title{
Mature $B$ cell acute lymphoblastic leukaemia with KMT2A-MLLT3 transcripts in children: three case reports and literature reviews
}

Yinghui Cui ${ }^{1,2,4,5}$, Min Zhou ${ }^{3}$, Pinli Zou ${ }^{1,4}$, Xin Liao ${ }^{1,5}$ and Jianwen Xiao ${ }^{1,2,4,5^{*}}$ (D)

\begin{abstract}
Background: Mature B cell acute lymphoblastic leukaemia (BAL) is characterised by French-American-British (FAB)L3 morphology and the presence of surface immunoglobulin (SlgM) light chain restriction. BAL is also considered as the leukaemic phase of Burkitt lymphoma (BL), in which $\mathrm{t}(8 ; 14)(\mathrm{q} 24$; q32) or its variants are related to the myelocytomatosis oncogene (MYC) rearrangement $(\mathrm{MYCr}$ ) is usually present. However, BAL with lysine methyltransferase $2 \mathrm{~A}$ (KMT2A, previously called Mixed lineage leukaemia, MLL) gene rearrangement (KMT2Ar, previously called MLLr) is rare.

Results: Three BAL patients with KMT2Ar were enrolled between January 2017 and November 2019, accounting for $1.37 \%$ of the B-ALL population in our hospital. We also reviewed 24 previously reported cases of BAL and KMT2Ar and analysed the features, treatment, and prognosis. Total 13 males and 14 females were enrolled in our research, and the average age at diagnosis was $19.5 \pm 4.95$ months old. In these 27 patients, renal, central nervous system (CNS) and skin involvement were existent in 6, 4 and 3 patients, respectively; 26 patients (26/27) showed non-ALL-L3 morphology, while one patient is ALL-L3; overexpression of CD19 was detected in most cases, negative or suspicious expression of CD20 was found in 64\% of patients. KMT2Ar was reported, but MYCr was not observed. 25 patients (25/27) achieved complete remission after chemotherapy or Stem cell transplantation. The patients were sensitive to chemotherapy, prospective event-free survival (pEFS) of BAL patients with KMT2Ar who received allogeneic haematopoietic stem cell transplantation (allo-HSCT) was higher than that in patients who received chemotherapy alone (83.33\% vs 41.91\%).

Conclusion: BAL patients with KMT2Ar had unique manifestations, including younger age at diagnosis and overexpression of CD19; expression of CD20 was rare, and MYCr was undetectable. The pEFS was higher in patients undergoing allo-HSCT than in patients undergoing chemotherapy alone.
\end{abstract}

Keywords: Mature B cell acute lymphoblastic leukaemia, KMT2A rearrangement, Children

\section{Introduction}

Acute lymphoblastic leukaemia (ALL) is the most common neoplasm in children, and B cell acute lymphoblastic leukaemia (B-ALL) accounts for $75-80 \%$ of all ALL cases

\footnotetext{
*Correspondence: tomahawk6502@sohu.com

${ }^{1}$ Division of Haematology and Oncology, Children's Hospital

of Chongqing Medical University, No. 136, Zhongshan 2nd Road, Yuzhong District, Chongqing 400014, People's Republic of China Full list of author information is available at the end of the article
}

[1]. According to the 2016 World Health Organization (WHO-2016) classification, B-ALL cases were classified into several subtypes: morphology, immunophenotype, cytogenetic, and molecular genetic characteristics [1,2]. For instance, the immunophenotypes of B-ALL populations were classified as precursor B-ALL (pB-ALL) and mature B-ALL (BAL) by flow cytometry (FCM) [2].

The most recurrent ALL type is $\mathrm{pB}-\mathrm{ALL}$. The $\mathrm{pB}-\mathrm{ALL}$ comprises $90 \%$ of B-ALL cases and is characterised by the morphologic type (French-American-British (FAB) 
classification systems) of ALL-L1 or ALL-L2; Flow cytometric analysis with combination of CD19, CyCD22, CyCD79a, TdT, HLA-DR and/or CD22, CD10, CD20, CyIgM, CD34 appear in pB-ALL [2-4]. Lysine methyltransferase 2A (KMT2A, previously called Mixed lineage leukaemia, MLL) gene rearrangements (KMT2Ar, previously called MLLr) are generally associated with ALL-L1/ALL-L2 pB-ALL and are present in $6 \%$ of paediatric ALL cases [2, 3]. KMT2A-AFF1 (also called MLLAF4) and KMT2A-MLLT3 (previously called MLL-AF9) fusion gene have been reported in ALL cases, while the KMT2A-MLLT3 fusion gene is a reverse factor of ALL cases $[4,5]$.

BAL is uncommon for ALL patients and is characterised by FAB-L3 morphology; the presence of surface IgM (sIgM) with light-chain restriction and the absence of immature B cell antigens is typical of BAL cases [2, 6]. BAL is often associated with the translocation $t(8 ; 14)$ (q24; 32 ) or its variants; the molecular genetics of BAL is characterised by myelocytomatosis oncogene (MYC) rearrangements (MYCr), and it is considered the leukaemic phase of Burkitt lymphoma (BL) [6, 7]. MYCr is overexpressed in more than $95 \%$ of BL/BAL patients [2].

However, rare cases of BAL with KMT2Ar expression have been reported in children and adults $[8,9]$. This study describes the clinical features, lab findings, treatment, and prognosis of three children with mature BAL with KMT2A-MLLT3 transcripts, and we also reviewed 24 case reports in the literature.

\section{Patients and methods Entry criteria}

1. One month old to under 18 years old;

2. Bone marrow morphology diagnosed as acute mature B lymphocytic leukaemia;

3. Immunological classification is acute mature B lymphocytic leukaemia;

4. with KMT2Ar.

\section{Exit criteria}

1. Acute lymphoblastic changes in chronic myeloid leukaemia;

2. Trisomy 21, or congenital or genetic disease accompanied by organ dysfunction;

3. Other secondary leukaemia;

4. Congenital immunodeficiency or metabolic disease;

5. Those who used glucocorticoids for 14 days or more within one month before enrollment or had any history of chemotherapy or radiotherapy within three months.

\section{Patients and samples collection}

Three BAL patients with KMT2Ar were enrolled in our hospital study. Clinical data, including age, gender, laboratory findings, treatment, and prognosis, were obtained from the patient records and retrospectively analysed. This study was approved by the Ethics Committee of the Children's Hospital of Chongqing Medical University (CHCMU) and Chengdu Women's \& Children's Central Hospital $(\mathrm{CWCCH})$, and written informed consent was obtained from all parents.

\section{Bone marrow analysis}

Three bone marrow (BM) samples were obtained at diagnosis and at different time points (TP) after chemotherapy. According to morphology, immunophenotype, cytogenetic and molecular genetics, classification was performed at diagnosis according to the WHO-2016 classification of tumours of the haematopoietic and lymphoid tissues [2]. The morphologic type was classified as ALL-L1, L2 or L3 by FAB subtyping. The immunophenotype was determined by FCM with monoclonal antibody markers consisting of B cell, T cell, myeloid and stem/ progenitor cell markers, and minimal residential disease (MRD) markers screened by FCM at diagnosis and monitored at different TP [1]. Chromosomal karyotyping and fluorescence in situ hybridisation (FISH) of ETV6RUNX1, BCR-ABL, KMT2Ar, PDGFRB and MYCr and other rearrangements were performed as reported in the literature; 29 common fusion genes (dupMLL, MLL/AF4, MLL/AF6, BCR/ABL1(p190), MLL/AF1P, MLL/AFX, ALL/ENL, BCR/ABL1(p210), TCF/PBX1, TEL/AML, SIL/TAL1, TLS/ERG, E2A/HLF, TEL/ABL1, HOX11, ETV6/ABL1, NUP214/ABL1, RANBP2/ABL1, SNX2/ ABL1, ZMIZ1/ABL1, RCSD1/ABL1, RCSD1/ABL2, ZC3HAV1/ABL2, PAG/ABL2, SSBP2/CSF1R, SSBP2/ PDGFR $\beta$, TNIP1TNIP1/ PDGFR $\beta$, ZEB2/PDGFR $\beta$, EBF1/PDGFR $\beta$ ) were assayed by multiplex nested reverse transcription-polymerase chain reaction (multiplex RTPCR) and confirmed by split RT-PCR as reported in the literature [10].

\section{Treatment}

Patient 1 and Patient 2 were treated according to the $\mathrm{B}$ non-Hodgkin lymphoma 2009 (B-NHL-2009) protocol for risk group 3 , modified according to the acute lymphoblastic leukaemia multicentre protocols (MCP-841, S1\& S2). Additional file 1: S3, S4 and S5 provide the risk group and drug dosage details. Patient 3 received chemotherapy according to the protocol of the Chinese Children's Cancer Group study ALL-2015 (CCCG-ALL-2015). Prophylactic intrathecal injections were administered for central nervous system (CNS) involvement (S6); BM samples 
were obtained and evaluated at different $\mathrm{TP}$ as protocols required. FCM monitored BM smears and MRD, and RTPCR was utilised to verify the results.

\section{Literature review}

Mature BAL patients with KMT2Ar in the literature were retrieved from PubMed, the Web of Science and China national knowledge infrastructure (CNKI). The keyword is "mature lymphocytic leukaemia" or "lymphoma" and "paediatrics". Data on clinical characteristics, laboratory findings, treatment, and prognosis were collected and analysed.

\section{Results}

\section{Clinical and lab findings}

A total of 198 newly diagnosed B-ALL patients, including 21 BAL patients, were admitted to CHCMU and CWCCH between January 2017 and November 2019, and $3 \mathrm{BAL}$ patients with MLL transcripts were identified, accounting for $1.37 \%$ of the B-ALL population. The clinical and laboratory findings for the three reported patients are shown in Table 1. BM samples were obtained at diagnosis, and the results of the BM examinations are listed in Table 2, Figs. 1 and 2.

\section{Treatment and prognosis}

Patient 1 and Patient 2 were treated with the B-NHL2009 protocol, and Patient 3 was treated with the CCCGALL-2015 protocol. They achieved complete remission (CR) according to morphological, FCM and molecular examination after one chemotherapy course. Allogenic haematopoietic stem cell transplantation (allo-HSCT) was considered by clinicians and was refused by the patients' parents. A total of six chemotherapy courses were completed for Patient 1 and Patient 2; as of the last follow-up in January 2020, these two patients had a CR status, and their event-free survival (EFS) times were 32 and 29 months, respectively. Chemotherapy was expected to continue for Patient 3, and the EFS time was three months.

\section{Literature review}

The literature, including Case reports and retrospective analysis, was searched in the abovementioned databases, and 12 articles involving 24 patients suffering from BAL with KMT2Ar were found. Clinical and laboratory findings, BM examination results, and the treatment and prognosis of these cases in the literature reports are listed in Tables 3,4 and $5[8,9,11-21]$.

Data from our hospital and literature about 27 patients suffering from BAL with KMT2Ar were collected and analysed. 13 males and 14 females were enrolled, and the average and the median age at diagnosis were $19.5 \pm 4.95$ months old and 12 months old, respectively (ranging from 6 weeks to 9 years); 14 (51.85\%) and $24(88.89 \%)$ patients were $\leq 1$ and $\leq 2$ years of age, respectively. Renal, testicular, $\mathrm{CNS}$ and skin involvement at diagnosis were present in 6, 1, 4 and 3 patients, respectively. The average white blood cell (WBC) and platelet (PLT) counts and haemoglobin $(\mathrm{Hb})$ levels were $87 \pm 35.24 \times 10^{9} / \mathrm{L}, \quad 69.96 \pm 5.38 \times 10^{9} / \mathrm{L}$ and $65.12 \pm 12.34 \mathrm{~g} / \mathrm{L}$, respectively.

Even though BAL is typically associated with the FABL3 morphology, in our research, $26(96.30 \%)$ of the 27 patients showed non-ALL-L3 morphology, and one patient presented with ALL-L3 morphology. FCM confirmed the mature B-ALL phenotype in all 27 patients. Expression of CD19, CD22, and sIgM with light-chain restriction was detected, and $\mathrm{TdT}$ and $\mathrm{CD} 34$ did not exist in most cases. Expression of CD20 was found in 25 patients. Interestingly, negative or suspicious expression of CD20 was found in 16 (64\%) patients, and positive expression of CD20 was detected with a monoclonal antibody in 9 (36\%) patients. Although 2 of 3 patients in our report presented with positive CD20 expression, the expression level of CD20 was lower than $30 \%$.

Chromosomal karyotype results were reported for 26 patients, while two patients had no metaphase chromosomes to be analysed; 11q23-related abnormal karyotypes were found in 11 patients. FISH of KMT2Ar was reported in 23 patients, and 22 (95.65\%) cases were positive. The results of the detection of KMT2Ar transcripts were presented for 16 patients. Fourteen patients were positive and were identified as having KMT2A-MLLT3 (Formerly MLL-AF9, 6 cases), KMT2A-MLLT5 (Formerly MLL-AF1, 2 cases), KMT2A-MLLT10 (Formerly MLL-AF10, 2 cases), and KMT2A-MLLT1(also called MLL-ENL, 2 cases), while

Table 1 Clinical and laboratory findings of reported patients

\begin{tabular}{|c|c|c|c|c|c|c|c|}
\hline $\mathrm{Pt}$ & Gender/age (m) & Clinical manifestations & WBC $\left(\times 10^{9} / \mathrm{L}\right)$ & $\mathrm{Hb}(\mathrm{g} / \mathrm{L})$ & PLT $\left(\times 10^{9} / \mathrm{L}\right)$ & Blast & LDH ( $\left.{ }^{U} / \mathrm{L}\right)$ \\
\hline 1 & Male/8 & Paleness and petechiae & 373.4 & 91 & 38 & 0.86 & 367 \\
\hline 2 & Male/24 & $\begin{array}{l}\text { Paleness, petechiae, hepatomegaly and abdominal lymphad- } \\
\text { enopathy }\end{array}$ & 92.05 & 53 & 168 & 0.90 & 598 \\
\hline 3 & Male/12 & $\begin{array}{l}\text { Fever, paleness, petechiae, hepatosplenomegaly, lymphadenop- } \\
\text { athy, parotid and renal involvement }\end{array}$ & 150.02 & 66 & 10 & 0.76 & 4059 \\
\hline
\end{tabular}




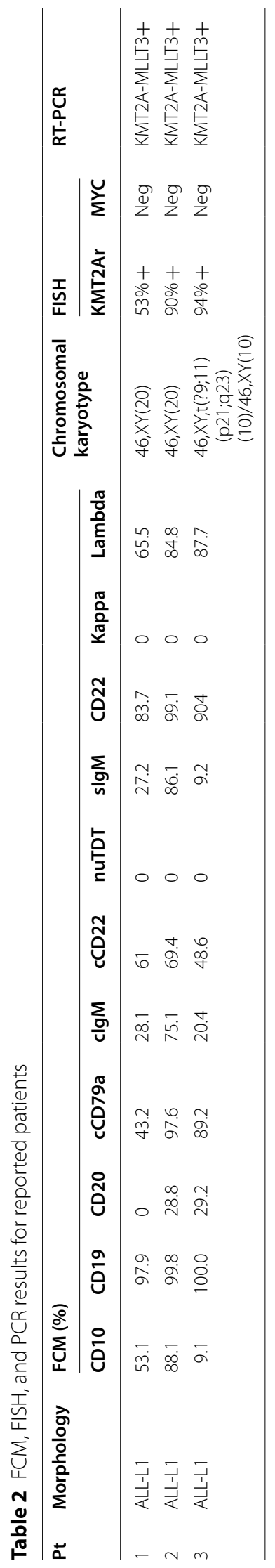




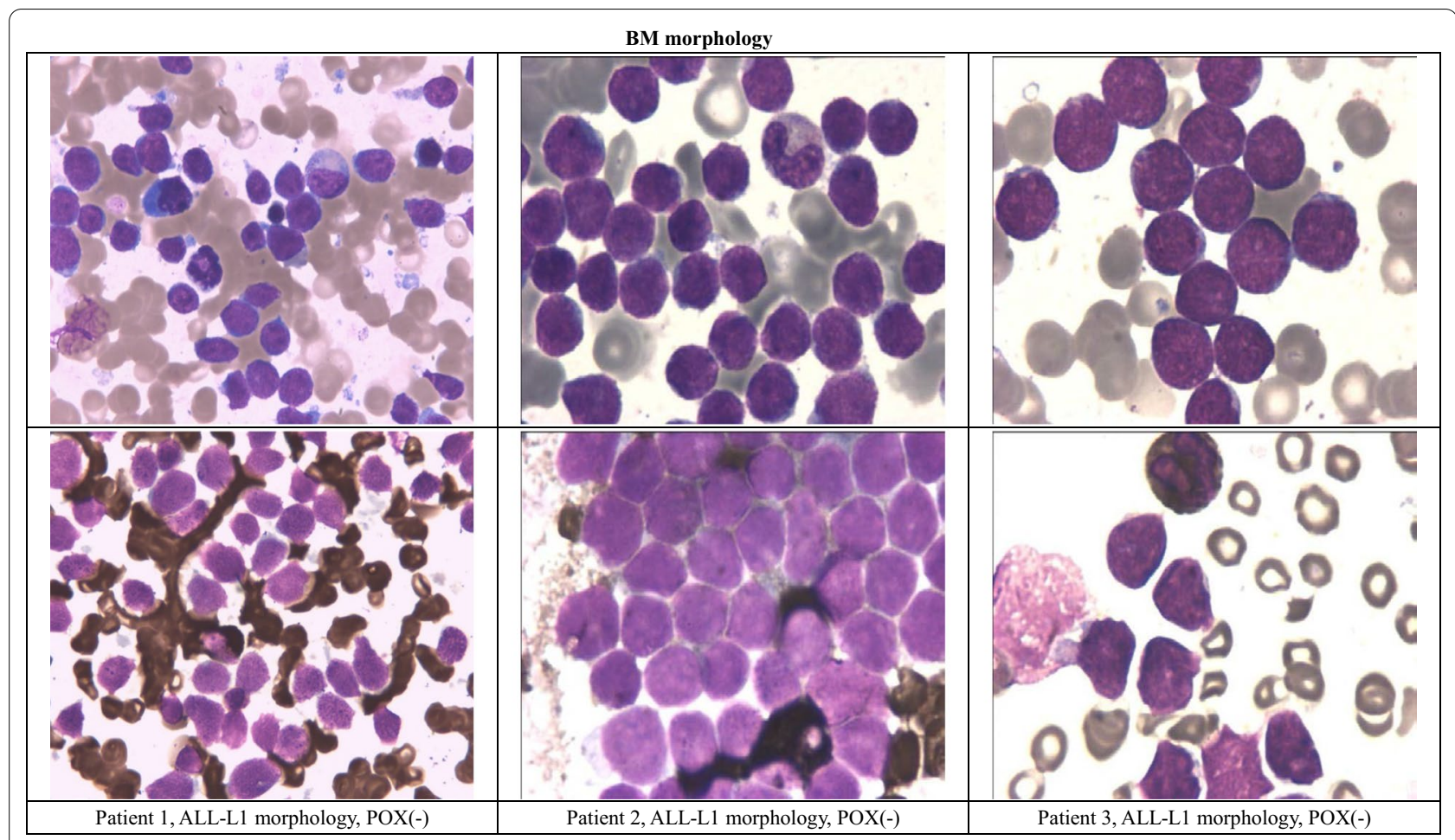

Fig. 1 BM morphology

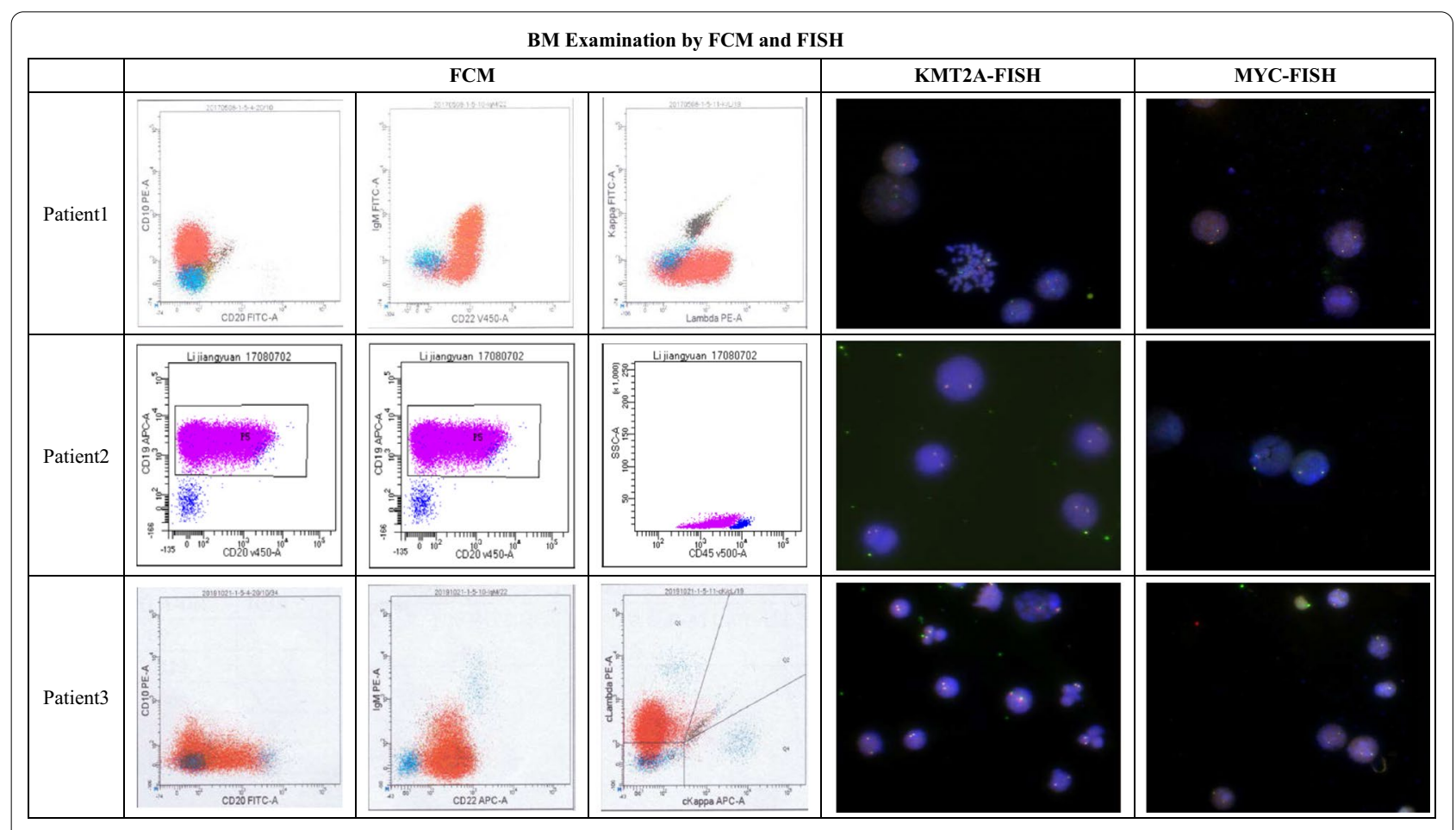

Fig. 2 BM examination by FCM and FISH 
Table 3 Clinical and laboratory findings of BAL with KMT2Ar according to the literature review

\begin{tabular}{|c|c|c|c|c|c|c|c|}
\hline $\mathrm{Pt}$ & Reference & Age (m) & Gender & Involved organs & WBC $\left(\times 10^{9} / L\right)$ & $\mathrm{Hb}(\mathrm{g} / \mathrm{L})$ & $\mathrm{PLT}\left(\times 10^{9} / \mathrm{L}\right)$ \\
\hline 1 & 13 & 96 & $\mathrm{~F}$ & Spleen & 43.9 & 80 & 6 \\
\hline 2 & 14 & 23 & $\mathrm{~F}$ & Liver, renal, CNS & 87 & 75 & 10 \\
\hline 3 & 15 & 13 & M & Lymph node, spleen & 60.1 & 83 & 63 \\
\hline 4 & 15 & 12 & $\mathrm{~F}$ & Lymph node, spleen, liver & 31.5 & 132 & 261 \\
\hline 5 & 17 & 11 & $\mathrm{~F}$ & - & 6.79 & 57 & 57 \\
\hline 6 & 16 & 4 & M & Skin, testicular & 33.2 & ND & 136 \\
\hline 7 & 16 & 8 & $\mathrm{~F}$ & Liver, spleen, adenopathy, skin & 160.9 & ND & 19 \\
\hline 8 & 18 & 23 & $\mathrm{~F}$ & Liver, CNS, renal & 87 & 75 & 10 \\
\hline 9 & 18 & 16 & $\mathrm{~F}$ & Spleen, CNS & 93.4 & 62 & 133 \\
\hline 10 & 18 & 5 & M & Renal, skin & 96.5 & 96 & 80 \\
\hline 11 & 18 & 13 & $\mathrm{~F}$ & Liver, spleen, lymph node & 24 & 49 & 10 \\
\hline 12 & 18 & 8 & M & spleen & 14.58 & 64 & 167 \\
\hline 13 & 19 & 1.5 & $\mathrm{~F}$ & Liver, spleen & 329 & ND & ND \\
\hline 14 & 20 & 4 & M & - & 117.2 & 94 & 54 \\
\hline 15 & 11 & 108 & M & Spleen & 1.8 & 72 & 45 \\
\hline 16 & 8 & 3 & M & Liver, spleen and renal & 857 & 88 & 46 \\
\hline 17 & 8 & 6 & M & Liver, spleen and renal, CNS & 119 & 89 & 85 \\
\hline 18 & 8 & 6 & M & Splenomegaly, skin & 11 & 72 & 104 \\
\hline 19 & 8 & 13 & $\mathrm{~F}$ & Lymph node & 39 & 45 & 11 \\
\hline 20 & 8 & 18 & $\mathrm{~F}$ & - & 68 & 3 & 16 \\
\hline 21 & 12 & 15 & $\mathrm{~F}$ & Liver, renal, lymph node & 54 & 78 & 41 \\
\hline 22 & 12 & 4 & $\mathrm{~F}$ & Liver, spleen & 295 & 29 & 45 \\
\hline 23 & 12 & 48 & $\mathrm{~F}$ & liver & 209 & ND & 21 \\
\hline 24 & 9 & 24 & M & liver, spleen, lymph node & 425.58 & 56 & 57 \\
\hline
\end{tabular}

F, female; $M$, male; ND, no data

one patient did not have an exact result. FISH may be the most accurate tool for the detection of KMT2Ar. The $t(8 ; 14)$ and its variant were not detected by karyotyping; FISH detected $\mathrm{MYCr}$ in 17 patients, and the results were negative.

The 27 patients who received chemotherapy included patients treated with ALL-like (12 cases), BL (8 cases) or Interfant-99 [21] (7 cases) protocols. One patient succumbed to sepsis, one patient presented with refractory status, 25 patients achieved CR, and the CR rate was $92.59 \%$. Besides, six patients received allo-HSCT, 1 (16.67\%) patient relapsed six months later, and the prospective 2-yr EFS (pEFS) was $83.33 \%$, as reported in the literature. Nineteen patients subsequently received chemotherapy according to the Interfant-99 (6 cases), BL (6 cases) or ALL-like (7 cases) protocols, and 9 (47.37\%) of them relapsed. The 2-yr pEFS was $41.91 \%$ (Fig. 3). Four patients in the Interfant-99 and BL groups relapsed, and the 2-yr pEFS in these groups were $40 \%$ and $33.33 \%$, respectively. One patient in the ALL-like group relapsed and died after five months of follow-up [22].

\section{Discussion}

BAL has been described as an uncommon subtype of B-ALL; it presents with a unique immunotype characterised by the expression of pan-B-cell markers (such as CD10, CD19, CD20, cCD79a.) and sIgM with light-chain restriction, whereas $\mathrm{pB}$-ALL with surface light-chain immunoglobulin restriction has also been reported [22, 23]. The clinical features, biological characteristics, treatment and prognosis of BAL are similar to those of $\mathrm{BL}$, since in the literature has $\mathrm{BL}$, we compared BAL and $\mathrm{BL}$ $[2,7]$. BAL patients often show an ALL-L3 FAB morphology; like BL, BAL is characterised by MYC translocations (chromosome 8q24) to an immunoglobulin gene locus, and the MYC gene overexpression was detected in most cases $[2,24]$. For patients treated with an intensive short course of chemotherapy, the EFS of BAL and BL has exceeded $90 \%$ [24].

KMT2A genes, lysine-specific methyltransferase $2 \mathrm{~A}$-related genes, occur in $2.5-5 \%$ of paediatric ALL patients and $70 \%$ of infant ALL patients [2]. KMT2Ar genes' presence is often correlated with the phenotype of $\mathrm{pB}$-ALL and leads to a worse prognosis $[1,2]$, but the 
Table 4 FAB type, FCM and chromosomal karyotyping of BAL with KMT2Ar according to the literature review

\begin{tabular}{|c|c|c|c|c|c|c|c|c|c|c|c|}
\hline $\mathrm{Pt}$ & FAB & Gender & CD19 & slgM & Light chain & CD10 & CD20 & CD22 & TdT & CD34 & Chromosomal karyotype \\
\hline 1 & L1 & $\mathrm{F}$ & Pos & Pos & L & Neg & Pos & ND & Pos & ND & $\begin{array}{c}46, X X(30) / 46, X X, 11, t(4, ?)(q 35 ; ?), t(9,11) \\
(p 22 ; q 23),+\operatorname{der}(I) t(1 ; 1)(q 21 ; q 14)(6)\end{array}$ \\
\hline 2 & L1 & $\mathrm{F}$ & + & Pos & L & Neg & Pos & Pos & ND & Neg & $46, \mathrm{XX}(4) / 46, \mathrm{XX}, \mathrm{t}(9 ; 11)(\mathrm{p} 21-22 ; \mathrm{q} 23)(12)$ \\
\hline 3 & L1 & M & + & Pos & $\mathrm{L}$ & Neg & $-/+$ & Pos & Neg & Neg & Normal \\
\hline 4 & L1 & $\mathrm{F}$ & + & Pos & L & Neg & $-/+$ & Pos & Neg & Neg & Normal \\
\hline 5 & Non-L3 & $\mathrm{F}$ & + & Pos & K & Neg & Pos & Pos & Neg & Neg & $46, X X(30)$ \\
\hline 6 & Non-L3 & M & + & Pos & L & Neg & $-/+$ & Pos & Neg & Neg & Normal \\
\hline 7 & Non-L3 & $\mathrm{F}$ & + & Pos & L & Neg & $-/+$ & Pos & Neg & Neg & Normal \\
\hline 8 & Non-L3 & $\mathrm{F}$ & + & Pos & L & Neg & $-/+$ & Pos & Neg & Neg & $46, X X[4] / 46, X X, t(9 ; 11)(p 21-22 ; q 23)[12]$ \\
\hline 9 & Non-L3 & $\mathrm{F}$ & + & Pos & K & Pos & $-1+$ & Pos & Neg & Neg & $46, X X[30]$ \\
\hline 10 & Non-L3 & M & + & Pos & $L$ & Neg & $-1+$ & Pos & Neg & Neg & 46,XX[20].ish add(11)(q23)[20 \\
\hline 11 & Non-L3 & $\mathrm{F}$ & + & Neg & $\mathrm{L}$ & Neg & ND & Pos & Neg & Neg & 46,XX[14].ish t $(9 ; 11)(p 22 ; q 23)$ \\
\hline 12 & Non-L3 & M & + & Pos & K & Neg & Neg & Pos & Neg & Neg & 46,XY[10].ish t(9;11)(p22;q23)[15] \\
\hline 13 & L1 & $\mathrm{F}$ & + & Pos & K & Pos & ND & ND & Neg & Neg & $46, X X$ \\
\hline 14 & L1 & M & + & Pos & Pos & Pos & Neg & Pos & Neg & Neg & $46, X Y[16] / 46, X X, t(9 ; 11 ; 11)(p 22 ; q 23 ; p 11.2)[4]$ \\
\hline 15 & ALL-L3 & M & $-1+$ & Pos & Pos & Pos & $-1+$ & Pos & $-/+$ & Neg & $46, X Y, t(11 ; 15)(q 23 ; q 15)[10] / 46, X Y[10]$ \\
\hline 16 & L1 & M & + & Pos & $\mathrm{L}$ & Neg & Pos & Pos & Neg & Pos & No metaphases \\
\hline 17 & Non-L3 & M & + & Pos & $\mathrm{L}$ & Pos & Pos & ND & Neg & Neg & $46, X Y, \operatorname{der}(2), t(10 ; 11)(p 12 ; q 23)[5]$ \\
\hline 18 & L1 & M & + & Pos & $L$ & Neg & Neg & Pos & Neg & Neg & $46-48, X Y,+11$, del(11)(q23), \\
\hline 19 & L1 & $\mathrm{F}$ & + & Pos & $L$ & Pos & Neg & Pos & Pos & Pos & $46, X X, \operatorname{add}(9)(p 24), \operatorname{ins}(10 ; 11)$ \\
\hline 20 & L1 & $\mathrm{F}$ & + & Pos & $\mathrm{L}$ & Pos & Neg & Pos & Pos & Pos & $46, X X[20]$ \\
\hline 21 & L1 & $\mathrm{F}$ & + & Pos & ND & Pos & Pos & ND & ND & Neg & No metaphases \\
\hline 22 & L1 & $\mathrm{F}$ & + & Pos & ND & Pos & Pos & Pos & Neg & Neg & Normal \\
\hline 23 & L1 & $\mathrm{F}$ & + & Pos & ND & Pos & Neg & Pos & ND & Neg & ND \\
\hline 24 & L2 & M & + & Pos & $L$ & Neg & Neg & ND & Neg & Neg & $46, X Y(3)$ \\
\hline
\end{tabular}

ND: No data; Pos: Positive; Neg: negative; L: Lambda chain; K: Kappa chain; Normal: reported normal chromosomal karyotype, but exact data were not presented

presence of KMT2Ar in BAL is a distinctive molecular biological feature, and patients' prognosis of KMT2Ar is unclear.

We reviewed the literature in databases, and a total of 27 patients, including the three patients described in our manuscript, were found. These three BAL patients with KMT2Ar have unique clinical manifestations and laboratory findings compared with $\mathrm{pB}-\mathrm{ALL}$ and $\mathrm{BL}$ patients. Infant leukaemia patients comprised half of these patients, and most of these patients $(24 / 27)$ were $\leq$ two years of age at onset, whereas the median ages of pB-ALL and BL patients were 9 and 2-5 years of age, respectively. Renal, CNS and skin involvement at diagnosis were not unusual and were present in 6,4 , and 3 cases, respectively, in patients with BAL with KMT2Ar; however, renal involvement is not rare in BAL, and CNS or skin involvement is uncommon in both pB-ALL and MAL [1, 6]. Although the reported patients were classified as having a BAL phenotype by FCM, overexpression of CD19 was detected in most cases, and expression of CD20 was not detected; nevertheless, coexpression of CD20 and CD19 is common in BL patients [2]. It has been revealed that rituximab [25], an anti-CD20 monoclonal antibody resulting in the selective depletion of $B$ lymphocytes, was unsuitable for treating these patients; any patient who appeared to be refractory or relapsed may benefit from chimeric antigen receptor $\mathrm{T}$ cell (CAR-T) immunotherapy targeting the overexpression of CD19 [26].

$\mathrm{BL}$ is often associated with $\mathrm{t}(8 ; 14)(\mathrm{q} 24 ; \mathrm{q} 32)$ or its variants, and MYCr is detectable in more than $95 \%$ of the BL population [2]. Translocation is the essential driver of the MYC gene's overexpression, and activation of the MYC gene leads to cell cycle progression, inhibition of differentiation, the promotion of cell proliferation and genomic instability and the activation of endogenous apoptotic programmes [25]. However, it is surprising that the MYC gene and its chromosomal translocation were undetectable in the MAL patients with KMT2Ar.

\section{Conclusions}

Standard treatment of BAL patients with KMT2Ar has not yet been established, and BAL patients seemed to be sensitive to chemotherapy, including chemotherapy administered according to the ALL, BL or Interfant-99 
Table 5 FISH, AB type, FCM and chromosomal karyotyping of BAL with KMT2Ar according to the literature review

\begin{tabular}{|c|c|c|c|c|c|c|c|c|}
\hline$\overline{\mathrm{Pt}}$ & KMT2A-FISH & MYC-FISH & Transcript $^{\mathrm{a}}$ & Protocol & Treatment response & HSCT & Relapse & EFS (m) \\
\hline 1 & ND & ND & ND & VLP & Refractory & Refractory & Refractory & 9 (OS) \\
\hline 2 & Pos & ND & $\mathrm{KMT} 2 \mathrm{~A}+$ & LMB89 & $C R$ & 0 & $\mathrm{BM}, \mathrm{CNS}$ & 9 \\
\hline 3 & Neg & Neg & ND & COG-ALL-1961 & $C R$ & No & No & 9 \\
\hline 4 & Pos & Neg & ND & CCG-ALL-1991 & $C R$ & No & No & 4 \\
\hline 5 & Pos & Neg & ND & Infant ALL & $C R$ & No & No & 4 \\
\hline 6 & Pos & Neg & ND & Interfant-99 & $C R$ & No & $\mathrm{BM}, \mathrm{TL}$ & 4 \\
\hline 7 & Pos & Neg & ND & Interfant-99 & $C R$ & 4/6 matched CBT & $\mathrm{BM}$ & 6 \\
\hline 8 & Pos & Neg & ND & LMB89 trial & $C R$ & No & $\mathrm{BM}, \mathrm{CNS}$ & 6 \\
\hline 9 & ND & Neg & ND & EORTC 02 & $C R$ & No & BM, CNS & 6 \\
\hline 10 & Pos & Neg & KMT2A-MLLT10 & Interfant-99 & $C R$ & No & No & 24 \\
\hline 11 & Pos & Neg & ND & FRALLE 2000 & $C R$ & No & No & 35 \\
\hline 12 & Pos & Neg & ND & Interfant-99 & $C R$ & No & $\mathrm{TL}$ & 14 \\
\hline 13 & Pos & ND & ND & ALL like & Died & died & died & 1.25 (OS) \\
\hline 14 & Pos & Neg & KMT2A-MLLT3 & ALL & $C R$ & No & No & 8 \\
\hline 15 & Neg & Neg & Neg & ALL & $C R$ & No & No & 48 \\
\hline 16 & Pos & ND & KMT2A/MLLT5 & Interfant-99 & $C R$ & No & $\mathrm{BM}, \mathrm{TL}$ & 4 \\
\hline 17 & Pos & ND & KMT2A-MLLT1 & BFM-2004 & $C R$ & No & $\mathrm{BM}, \mathrm{TL}$ & 19 \\
\hline 18 & Pos & ND & KMT2A-MLLT1 & Interfant-99 & $C R$ & No & BM & 21 \\
\hline 19 & Pos & ND & KMT2A-MLLT10 & Interfant-99 & $C R$ & No & No & 32 \\
\hline 20 & Pos & ND & KMT2A-MLLT5 & ALLIC-09 & $C R$ & No & No & 21 \\
\hline 21 & Pos & ND & KMT2A-MLLT3 & BFM like & $C R$ & Sible-HSCT & No & 108 \\
\hline 22 & Pos & Neg & KMT2A-MLLT3 & KMT2A03 & $C R$ & mismatched-CBT & No & 72 \\
\hline 23 & ND & ND & KMT2A-MLLT3 & BFM-95 & $C R$ & MUD-CBT & No & 12 \\
\hline 24 & ND & Neg & KMT2A-MLLT3 & B-NHL-2010 & $C R$ & No & CNS & 13 \\
\hline
\end{tabular}

ND, no data; Pos, positive; Neg: negative; HSCT, haematopoietic stem cell transplantation; CBT, cord blood transplant; MUD, matched unrelated donor; BM, bone marrow; TL, testicular leukaemia; CNS, central nervous system; EFS, event-free survival; OS, overall survival

${ }^{a}$ Transcripts of KMT2Ar were detected by RT-PCR except for patient 2, who was tested by Northern Blot

protocols (The details are in the Additional file 1). Most of these patients achieved CR after receiving one chemotherapy course, but the prognosis of patients subjected to different treatments was widely divergent. The pEFS was higher in the allo-HSCT group than in the chemotherapy

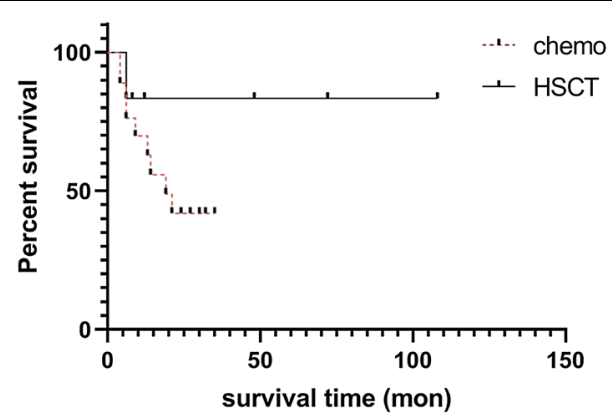

Fig. 3 Survival curves of patients. Chemo, chemotherapy; HSCT, haematopoietic stem cell transplantation. Survival analysis by the Kaplan-Meier method and the survival curve of the whole group. Prospective event-free survival (pEFS) in patients who received allogeneic haematopoietic stem cell transplantation (allo-HSCT) was higher than that in patients who received chemotherapy alone (83.33\% vs $41.91 \%)$ group. Even though two patients described in our report received chemotherapy with the $\mathrm{BL}$ protocol and survived more than two years, the prognosis of patients treated with chemotherapy has remained poor, and alloHSCT should be recommended for patients with CR1 status.

\footnotetext{
Abbreviations

AFF1: Proteini AF4/FMR2 family member 1 Gene; ALL: Acute lymphoblastic leukemia; allo-HSCT: Allogeneic haematopoietic stem cell transplantation; BAL: Mature B cell acute lymphoblastic leukaemia; B-ALL: B cell acute lymphoblastic leukaemia; BL: Burkitt lymphoma; BM: Bone marrow; B-NHL-2009: B non-Hodgkin lymphoma 2009 protocol; CAR-T: Chimeric antigen receptor T cell; CCCG-ALL-2015: Chinese Children's Cancer Group study ALL-2015; CD: Cluster of differentiation; CHCMU: Children's Hospital of Chongqing Medical University; CNKI: China national knowledge infrastructure; CNS: Central nervous system; CR: Complete remission; CWCCH: Chengdu Women's \& Children's Central Hospital; FAB: French-American-British; FCM: Flow cytometry; FISH: Fluorescence in situ hybridisation; Hb: Haemoglobin; KMT2Ar: KMT2A rearrangement; MCP-841: Acute lymphoblastic leukaemia multicentre protocol; MRD: Minimal residential disease; multiplex RT-PCR: Multiplex nested reverse transcription-polymerase chain reaction; MYC: Myelocytomatosis oncogene; MYCr: MYC rearrangement; KMT2A: Lysine methyltransferase 2A; pB-ALL: Precursor B-ALL; pEFS: Prospective event-free survival; PLT: Platelet; slgM: Surface immunoglobulin; TP: Time point; WBC: White blood cell; WHO: World Health Organization.
} 


\section{Supplementary Information}

The online version contains supplementary material available at https://doi. org/10.1186/s13023-021-01972-5.

Additional file 1: Describe the details of the treatment, include $\mathbf{S 1}$ : inclusion and exclusion criteria of the B-NHL-2009 protocol; S2: the staging system of the B-NHL-2009 protocol; S3: the risk groups of the B-NHL-2009 protocol; S4: treatment planning of the B-NHL-2009 protocol; S5: schedule of the B-NHL-2009 protocol; S6: schedule of intrathecal injections for CNS involvement.

\section{Acknowledgements}

We are grateful to the patients' families for their participation and support of this study.

\section{Authors' contributions}

$Y C$ contributed to collecting data, performed the revision and the interpretation of all the clinical data and wrote the manuscript. MZ, PZ and XL contributed to collecting data. JX conceived the study and participated in its design and coordination, and helped draft the manuscript. All authors read and approved the final manuscript.

\section{Funding}

This study was supported by the Chongqing Science and Technology Commission of PR China (project no. cstc2018jsyj-jsyjX0015).

\section{Availability of data and materials}

The data, including the clinical and laboratory findings and treatment and prognosis data, are listed in the article and the Additional file 1.

\section{Declarations}

\section{Ethics approval and consent to participate}

The study protocol was approved on 22 April 2015 by the institutional ethics committee (Institutional Review Board of Children's Hospital of Chongqing Medical University). The study was registered the 4 June 2014 and the 22 May 2018 under the Chinese Clinical Trial Registry identifier ChiCTR-IPR-14005706 and ChiCTR1900025690(http://www.chictr.org.cn/showproj.aspx?proj=10115 \& http://www.chictr.org.cn/showproj.aspx?proj=27848).

\section{Consent for publication}

The patients in this manuscript have given written informed consent to the publication of their case details.

\section{Competing interests}

The authors declare that they have no competing interests.

\section{Author details \\ 'Division of Haematology and Oncology, Children's Hospital of Chongqing Medical University, No. 136, Zhongshan 2nd Road, Yuzhong District, Chong- qing 400014, People's Republic of China. ${ }^{2}$ Ministry of Education Key Labora- tory of Child Development and Disorders, Chongqing, People's Republic of China. ${ }^{3}$ Department of Hematology, Chengdu Women's \& Children's Central Hospital, Chengdu, People's Republic of China. ${ }^{4}$ National Clinical Research Center for Child Health and Disorders, Chongqing, People's Republic of China. ${ }^{5}$ Chongqing Key Laboratory of Paediatrics, Chongqing, People's Republic of China.}

Received: 21 February 2021 Accepted: 19 July 2021

Published online: 30 July 2021

\section{References}

1. Cui L, Li Z-G, Chai Y-H, Yu J, Gao J, Zhu X-F, et al. Outcome of children with newly diagnosed acute lymphoblastic leukemia treated with CCLG-ALL 2008: the first nation-wide prospective multicenter study in China. Am J Hematol. 2018;93:913-20. https://doi.org/10.1002/ajh.25124.
2. Swerdlow SH, Campo E, Harris NL, Jaffe ES, Pileri SA, Stein H, et al., editors. WHO Classification of Tumours of Haematopoietic and Lymphoid Tissues. Revised 4th ed. Lyon: International Agency for Research on Cancer (IARC) 69372 Lyon Cedex 08, France; 2017.

3. Wenzinger C, Williams E, Gru AA. Updates in the pathology of precursor lymphoid neoplasms in the revised fourth edition of the WHO classification of tumors of hematopoietic and lymphoid tissues. Curr Hematol Malig Rep. 2018;13:275-88. https://doi.org/10.1007/s1 1899-018-0456-8.

4. Burmeister T, Meyer C, Gröger D, Hofmann J, Marschalek R. Evidencebased RT-PCR methods for the detection of the 8 most common MLL aberrations in acute leukemias. Leuk Res. 2015;39:242-7. https://doi.org/ 10.1016/j.leukres.2014.11.017.

5. Yang L, Ding L, Liang J, Chen J, Tang Y, Xue H, et al. Relatively favorable prognosis for MLL-rearranged childhood acute leukemia with reciprocal translocations. Pediatr Blood Cancer. 2018;65: e27266. https://doi.org/10. 1002/pbc.27266.

6. Hoelzer D, Walewski J, Döhner H, Viardot A, Hiddemann W, Spiekermann $\mathrm{K}$, et al. Improved outcome of adult Burkitt lymphoma/leukemia with rituximab and chemotherapy: report of a large prospective multicenter trial. Blood. 2014;124:3870-9.

7. Song JY, Venkataraman G, Fedoriw Y, Herrera AF, Siddiqi T, Alikhan MB, et al. Burkitt leukemia limited to the bone marrow has a better prognosis than Burkitt lymphoma with bone marrow involvement in adults. Leuk Lymphoma. 2016;57:866-71. https://doi.org/10.3109/10428194.2015. 1085529.

8. Sajaroff EO, Mansini A, Rubio P, Alonso CN, Gallego MS, Coccé MC, et al. B-cell acute lymphoblastic leukemia with mature phenotype and MLL rearrangement: report of five new cases and review of the literature. Leuk Lymphoma. 2016;57:2289-97. https://doi.org/10.3109/10428194.2016. 1141407.

9. Yao QH, Liu YF, Fang YQ, Zhao XM. [Childhood B-cell acute lymphoblastic leukemia of nonL3 morphology with mature phenotype and MLL-AF9 gene fusion: a case report and literatures review]. Zhonghua Xue Ye Xue Za Zhi. 2018;39:947-9.

10. Pallisgaard N, Hokland P, Riishøj DC, Pedersen B, Jørgensen P. Multiplex reverse transcription-polymerase chain reaction for simultaneous screening of 29 translocations and chromosomal aberrations in acute leukemia. Blood. 1998:92:574-88.

11. Smith MC, Kressin MK, Crawford E, Wang XJ, Kim AS. B Lymphoblastic leukemia with a novel $\mathrm{t}(11 ; 15)$ (q23;q15) and unique Burkittoid morphologic and immunophenotypic findings in a 9-year-old boy. Lab Med. 2015;46:320-6. https://doi.org/10.1309/LMOBOC84GSQGHYKD.

12. Sarashina T, Iwabuchi H, Miyagawa N, Sekimizu M, Yokosuka T, Fukuda K, et al. Hematopoietic stem cell transplantation for pediatric mature B-cell acute lymphoblastic leukemia with non-L3 morphology and MLL-AF9 gene fusion: three case reports and review of the literature. Int J Hematol. 2016;104:139-43. https://doi.org/10.1007/s12185-016-1971-9.

13. Lorenzana AN, Rubin CM, Le Beau MM, Nachman J, Connolly P, Subramanian $\mathrm{U}$, et al. Immunoglobulin gene rearrangements in acute lymphoblastic leukemia with the 9;11 translocation. Genes Chromosom Cancer. 1991;3:74-7. https://doi.org/10.1002/gcc.2870030113.

14. Talmant P, Berger R, Robillard N, Mechineau-Lacroix F, Garand R. Childhood B-cell acute lymphoblastic leukemia with FAB-L1 morphology and a t $(9 ; 11)$ translocation involving the MLL gene. Hematol Cell Ther. 1996;38:265-8. https://doi.org/10.1007/s00282-996-0265-8.

15. Li S, Lew G. Is B-lineage acute lymphoblastic leukemia with a mature phenotype and 11 morphology a precursor B-lymphoblastic leukemia/ lymphoma or Burkitt leukemia/lymphoma? Arch Pathol Lab Med. 2003;127:1340-4

16. Tsao L, Draoua HY, Osunkwo I, Nandula SV, Murty VVS, Mansukhani M, et al. Mature B-cell acute lymphoblastic leukemia with $\mathrm{t}(9 ; 11)$ translocation: a distinct subset of B-cell acute lymphoblastic leukemia. Mod Pathol. 2004;17:832-9.

17. Frater JL, Batanian JR, O'Connor DM, Grosso LE. Lymphoblastic Leukemia With Mature B-Cell Phenotype in Infancy. J Pediatr Hematol Oncol. 2004 Oct;26(10):672-7. https://doi.org/10.1097/01.mph.0000142493.56793.

18. Blin N, Méchinaud F, Talmant P, Garand R, Boutard P, Dastugue N, et al. Mature B-cell lymphoblastic leukemia with MLL rearrangement: an uncommon and distinct subset of childhood acute leukemia. Leukemia. 2008;22:1056-9. 
19. Lim L, Chen K-S, Krishnan S, Gole L, Ariffin H. Mature B-cell acute lymphoblastic leukaemia associated with a rare MLL-FOXO4 fusion gene. $\mathrm{Br} J$ Haematol. 2012;157:651-651. https://doi.org/10.1111/j.1365-2141.2012. 09091.x.

20. Kim B, Lee S-T, Kim H-J, Lee S-H, HeeYoo K, Koo HH, et al. Acute lymphoblastic leukemia with mature B-cell phenotype and $t(9 ; 11 ; 11)$ (p22;q23;p11.2): a case study and literature review. Ann Lab Med. 2014;34:166-9. https://doi.org/10.3343/alm.2014.34.2.166.

21. Pieters R, Schrappe M, De Lorenzo P, Hann I, De Rossi G, Felice M, et al. A treatment protocol for infants younger than 1 year with acute lymphoblastic leukaemia (Interfant-99): an observational study and a multicentre randomised trial. Lancet. 2007;370:240-50.

22. Worch J, Rohde M, Burkhardt B. Mature B-cell lymphoma and leukemia in children and adolescents — review of standard chemotherapy regimen and perspectives. Pediatr Hematol Oncol. 2013;30:465-83. https://doi. org/10.3109/08880018.2013.783891.

23. Kansal R, Deeb G, Barcos M, Wetzler M, Brecher ML, Block AW, et al. Precursor B lymphoblastic leukemia with surface light chain immunoglobulin restriction. Am J Clin Pathol. 2004;121:512-25. https://doi.org/10.1309/ WTXCQ5NRACVXTYBY.

24. Chang VY, Basso G, Sakamoto KM, Nelson SF. Identification of somatic and germline mutations using whole exome sequencing of congenital acute lymphoblastic leukemia. BMC Cancer. 2013;13:55. https://doi.org/10. 1186/1471-2407-13-55.

25. Magrath I, Adde M, Shad A, Venzon D, Seibel N, Gootenberg J, et al. Adults and children with small non-cleaved-cell lymphoma have a similar excellent outcome when treated with the same chemotherapy regimen. J Clin Oncol. 1996;14:925-34. https://doi.org/10.1200/JCO.1996.14.3.925.

26. Abramson JS. Anti-CD19 CART-cell therapy for B-cell non-hodgkin lymphoma. Transfus Med Rev. 2020;34:29-33. https://doi.org/10.1016/j.tmrv. 2019.08.003.

\section{Publisher's Note}

Springer Nature remains neutral with regard to jurisdictional claims in published maps and institutional affiliations.
Ready to submit your research? Choose BMC and benefit from:

- fast, convenient online submission

- thorough peer review by experienced researchers in your field

- rapid publication on acceptance

- support for research data, including large and complex data types

- gold Open Access which fosters wider collaboration and increased citations

- maximum visibility for your research: over $100 \mathrm{M}$ website views per year

At BMC, research is always in progress.

Learn more biomedcentral.com/submissions 